\title{
SARS-CoV-2 outbreak in a Canadian suburban tertiary hospital necessitating full facility closure: a descriptive observational study
}

\author{
Jamil N. Kanji MD, Y.L. Elaine Chan MPH, Lesia R. Boychuk MD, Curtiss Boyington MD, \\ Sebora Turay MSc, Melissa Kobelsky BScN, Carolyn Doroshuk BScN, Philana Choo BSc BScN, \\ Susan Jacka BScN MN, Erin Roberts BScN MBA, Karen Leighton BScN, Stephanie W. Smith MD MSc, \\ Christopher Sikora MD MPH, Robert Black MD
}

\section{Abstract}

Background: SARS-CoV-2 can cause outbreaks in community- and hospital-based settings. The aim of this study was to provide a detailed epidemiologic account of a hospital-wide SARS-CoV-2 outbreak and provide a description of case evaluations, transmission networks and the interventions implemented to stem the outbreak.

Methods: We conducted a retrospective descriptive study of a hospital-wide SARS-CoV-2 outbreak at the Misericordia Community Hospital (Edmonton) from June 21 to Aug. 14, 2020. We reviewed hospital chart, public health and occupational health records to determine demographics, case type (community- or hospital-acquired), need for critical care and outcome for each case linked to the outbreak (patients, hospital staff, and community and patient visitors). We developed detailed transmission networks using epidemiologic data to determine what variables may have contributed to transmission.

Results: Fifty-eight cases of SARS-CoV-2 infection were linked to this hospital outbreak (31 patients, 25 staff members and 2 visitors; $66 \%$ female, age range 19-97 years). One patient required critical care, and 11 deaths were recorded (all among inpatients). Most cases were hospital-acquired $(91 \%)$, and $28 \%$ were asymptomatic at the time of diagnosis. The outbreak was composed of 2 clusters driven by protective equipment breaches, premature removal of precautions, transmission in small staff quarters and infection of a staff member after exposure to a wandering patient with dementia and asymptomatic, undetected SARS-CoV-2 infection.

Interpretation: A detailed epidemiologic review of this hospital-wide outbreak shows that a SARS-CoV-2 outbreak can involve complex transmission chains and clusters. Multipronged bundled approaches, aggressive contact tracing, and patient and staff prevalence screening are important to help bring such outbreaks under control, along with ongoing vigilance in detecting delayed cases.

pread of SARS-CoV-2 (the virus that causes COVID-19) between individuals is much higher than with other common respiratory viruses. ${ }^{1}$ This is best illustrated by the fact that SARS-CoV-2 has a reproduction rate of 3 to $3.32 .{ }^{1}$ Over the course of the pandemic (March 2020 to December 2021), the COVID-19 reproduction rate in Canada has ranged from 0.67 to 2.37 (www.theglobaleconomy.com/Canada/covid_ reproduction_rate/). During the early part of the pandemic, data from China reported nosocomial spread in up to $44 \%$ of cases. ${ }^{2}$ Hospital-acquired SARS-CoV-2 infection poses an immense challenge for infection prevention and control (IPC) and occupational health and safety (OHS) teams, and has been associated with patient mortality as high as $29 \%$ to $37 \% .^{3-5}$

The spread of SARS-CoV-2 in health care facilities has often led to staff shortages. Depending on the study, health care worker infection rates range from $1 \%$ to $20 \%{ }^{6-8}$ Remaining health care workers work longer hours and more frequent shifts, and they have higher patient loads. ${ }^{9}$ Facility-wide SARS-CoV-2 outbreaks that have resulted in full hospital closure are uncommon; they have been reported to date in 2 other hospitals, one in Germany, and another in Thailand..$^{10,11}$ Although hospital closures are temporary, their impact - leading to abrupt cessation of medical services in the area the hospital serves - can be immense, including strain on emergency services in neighbouring areas, relocation of patients booked for emergency procedures, and cancellation and delay of elective surgical procedures and imaging tests, as well as negative outcomes for medical education in academic centres and hospital employee well-being. ${ }^{12,13}$

Competing interests: None declared.

This article has been peer reviewed.

Correspondence to: Jamil Kanji, jamil.kanji@ahs.ca CMAJ Open 2022 February 22. DOI:10.9778/cmajo.20210064 
From June to August 2020, a facility-wide COVID-19 outbreak occurred in our tertiary care suburban hospital. The extent of the outbreak and the number of contacts who required isolation (including staff) led to the decision to close the hospital to new admissions and transfers, and to cease all in-person ambulatory appointments. This was the only way to control the outbreak and continue to provide services safely for admitted patients. The aim of this study was to provide a detailed retrospective epidemiologic description of the outbreak, an analysis of the patterns of spread, and identification of key interventions used for control of a hospitalwide outbreak.

\section{Methods}

\section{Study design}

We conducted a retrospective epidemiologic study to describe a facility-wide COVID-19 outbreak involving admitted patients, staff and patient contacts at the Misericordia Community Hospital (Edmonton) that occurred between June 21, 2020, and Aug. 14, 2020.

\section{Definitions}

A confirmed case of SARS-CoV-2 infection was any person who tested positive for the presence of SARS-CoV-2 RNA on a nasopharyngeal swab or other deep respiratory specimen (endotracheal aspirate or bronchoalveolar lavage) using a real-time, reverse-transcriptase polymerase chain reaction (rRT-PCR) assay. Testing was conducted at the public health laboratory (Alberta Precision Laboratories), which used an rRT-PCR that detected the envelope gene of SARS-CoV-2 (approximate sensitivity of $91 \%$ ). ${ }^{14-16}$ Based on a median incubation period of 4 to 6 days, ${ }^{17}$ community-acquired and hospital-acquired infections were defined as a confirmed case of SARS-CoV-2 infection within 7 days and more than 7 days postadmission, respectively. ${ }^{18}$ All definitions were aligned with provincial standards developed by the Alberta Health Services (AHS) IPC surveillance team, which are based on definitions from Alberta Health and the Public Health Agency of Canada. ${ }^{18-20}$

\section{Setting and population}

The Misericordia Community Hospital is a 312-bed community teaching hospital built in 1969, which provides 24-hour emergency department services, adult medical and surgical services (including cardiac and critical care), obstetrical services, geriatric services and acute mental health services. The hospital does not provide pediatric services, except for neonatal intensive care. Excluding critical care units, $51 \%$ of patient care beds are 4-bed rooms, 35\% are 2-bed rooms and $14 \%$ are single rooms. Multi-bed rooms have curtain barriers between patients and 1 shared bathroom. In 2-bed rooms, beds are spaced approximately $1.5 \mathrm{~m}$ apart; in 4-bed rooms, beds are spaced approximately $1.5 \mathrm{~m}$ apart (next to each other) and $2.5 \mathrm{~m}$ across from each other.

Staff members included in this study were all those who were working at the facility during the time of outbreak - all ward-based staff (porters, food and environmental services workers, nurses and allied health care providers, trainees of all types, physicians, and facility-maintenance workers who conducted work on affected units). Designated patient visitors, household family members and nonhospital staff contacts of patient and staff cases of SARS-CoV-2 infection were included if they were considered to be exposed to a hospitalacquired case based on contact tracing conducted by hospital IPC staff or AHS Public Health.

\section{Hospital procedures for COVID-19}

\section{Pandemic preparedness measures}

In January and February 2020, the IPC section of the provincial health authority (AHS) developed standardized protocols for COVID-19 symptom screening, testing, personal protective equipment (PPE), continuous masking in patient care areas and related isolation protocols. Our hospital implemented these protocols, and hospital-based infection control professionals provided training to unit managers, who then provided education to patient care area staff. Hospital management organized online sessions and webinars, and made mandatory online training modules available for all health care staff. The IPC section of the hospital provided multiple live town-hall forums via video conference platforms to help answer questions and provide on-demand teaching based on concerns raised.

During this time, the hospital implemented a reduction in elective surgeries, a transition to online and telephone appointments for ambulatory patients and back-up staffing protocols to prepare for an influx of patients with COVID-19, in collaboration with AHS. Most protocols were fully implemented by the end of February 2020, as the first COVID-19 cases were diagnosed across Canada. At the same time, hospital volunteer services were discontinued for pandemic preparation purposes.

\section{IPC procedures and contact tracing}

Alberta Health Services recommended testing for SARS$\mathrm{CoV}-2$ for all who experienced core or expanded symptoms, using an acute care algorithm for assessment. ${ }^{21}$ Patients with suspected or confirmed COVID-19 were placed on contact and droplet precautions pending test results. Contact and droplet precautions required all those entering the room to don a medical-grade surgical mask, eye protection, a protective gown and medical gloves (as per guidance from the Public Health Agency of Canada at the time)..$^{22}$ If an aerosolgenerating medical procedure was being conducted, a fitted N95 mask was required in place of a surgical mask. ${ }^{23} \mathrm{~A}$ list of procedures that met the definition of an aerosol-generating medical procedure ${ }^{23}$ was posted on the AHS website for health care workers to consult.

Isolation precautions were required to be maintained for 14 days after the date of symptom onset in those who tested positive for SARS-CoV-2, and for 21 days in patients who were severely immunocompromised or required critical care. ${ }^{24}$ Health care staff were permitted to use fitted N95 
masks outside of aerosol-generating medical procedures for any COVID-19 patient if a staff member determined that they were at high risk of exposure based on a point-of-care risk assessment. ${ }^{25}$ Physicians were urged to retest any symptomatic patients who tested negative for SARS-CoV infection in 48 hours if there was no alternate diagnosis after clinical re-evaluation. ${ }^{26}$

Confirmed symptomatic cases were considered contagious 48 hours before the date of symptom onset. Staff were considered close contacts if they were not wearing appropriate PPE, if they experienced a PPE breach for 15 minutes or more, or if they did not have appropriate PPE in a small work space with a confirmed case where social distancing could not occur.

Every confirmed patient or staff case of COVID-19 underwent investigation to trace contacts, determine potential exposures and identify a date of symptom onset (via contact tracing protocols designed by AHS IPC and AHS Public Health). Contact tracing for hospitalized outbreak cases was conducted by hospital-based infection control professionals in collaboration with AHS Public Health. For hospital staff, hospital OHS used a provincially developed contact tracing questionnaire. AHS Public Health assisted with contact tracing and follow-up with community contacts of outbreak-related cases (including visitors and family members of patients, as well as family members of hospital staff), and of patients identified as outbreak contacts who were discharged to the community or other facilities.

All contacts identified by AHS Public Health to have had an exposure were advised to quarantine for 14 days in the community and followed for the development of symptoms and testing. Contacts who were admitted or transferred to other health care facilities were placed on empiric contact and droplet precautions for 14 days, with twice-daily symptom monitoring. The duration of isolation precautions for contacts began from the day the person last had contact with the known contagious COVID-19 case or 14 days from the date of symptom onset if they developed COVID-19.

From a facility perspective, continuous masking for patients when they were out of their rooms or off the unit (e.g., for radiology procedures or other investigations) was implemented on May 21, 2021.

\section{Epidemiologic investigation}

On June 21, 2020, a unit-based COVID-19 outbreak was identified after confirmation of 2 COVID-19 cases with no clear epidemiologic links. A hospital outbreak response team was formed that included membership from the hospital's IPC, OHS, department managers and site leadership, AHS Public Health and an epidemiologist (Y.L.E.C.) from our provincial health authority incident command structure. ${ }^{27}$ Because of ongoing transmission, a facility-wide outbreak was declared on July 8, 2020, and the hospital was closed to new admissions; all ambulatory visits and elective procedures were cancelled or rescheduled at other hospitals. The outbreak was declared over after no further patient or staff cases had been detected for 2 incubation periods ( $28 \mathrm{~d}$ ) from the last known onsite exposure; the hospital reopened on Aug. 14, 2020.

\section{Initial outbreak unit procedures}

From the beginning of the COVID-19 pandemic, any hospital unit found to have a case of hospital-acquired SARS$\mathrm{CoV}-2$ infection was placed on outbreak procedures and closed to admissions. Baseline unit prevalence testing for SARS-CoV-2 infection was conducted on all patients to assess for undetected spread (repeated every 72 hours for 14 days). Voluntary testing every 5 days was recommended for asymptomatic unit staff. This recommendation was communicated by outbreak notification emails to all unit staff, and testing was available at the hospital to optimize the number of staff members who could be tested. Staff who became symptomatic at any time during their shift were immediately advised to leave work, self-isolate and arrange COVID-19 testing. Health care workers on an outbreak unit were cohorted whenever possible, IPC signage was increased, cleaning with virucidal products was enhanced to multiple times daily, and all patients were placed on contact and droplet precautions for the duration of the outbreak.

\section{Additional outbreak interventions}

During the course of the outbreak, the outbreak response team implemented a series of patient and staff interventions aimed at controlling the spread of SARS-CoV-2 (Table 1). Further intervention details are outlined in Appendix 1, Methods S1, available at www.cmajopen.ca/content/10/1/E137/suppl/DC1.

\section{Outcomes}

The primary outcomes for each case of COVID-19 attributable to this outbreak were as follows: community-acquired or hospital-acquired determination, admission to a critical care unit, and death. A secondary outcome was positivity rates of SARS-CoV-2 prevalence screening among patients and asymptomatic staff members.

\section{Data sources}

Demographic and case data were based on a review of hospital paper charts, hospital electronic medical records (Epic EMR; Epic) and the AHS IPC provincial surveillance database (ProvSurv). ${ }^{18}$ This data extraction was carried out by L.R.B., C.B., S.T., M.K., C.D., P.C., S.J. and K.L. Information for COVID-19 cases and contacts outside the hospital was obtained from relevant hospital unit patient visitor logs (physical sign-in/-out books) and the AHS Public Health communicable disease and surveillance record database. Visitors were not permitted to visit family members in hospitals until they had signed a visitor log. Numbers and testing results for staff screening were provided in aggregate form by the OHS team, to which all staff results were reported.

\section{Statistical analysis}

Epidemiological curves were created by Y.L.E.C. using Excel 2016 (Microsoft Corp.). We used information from hospital records and independent contact tracing to determine contacts and potential transmission between cases. We explored and visualized transmission networks using the ForceAtlas2 algorithm of Gephi (version 0.9.2). ${ }^{28,29}$ 
Table 1: Interventions implemented by the outbreak response team

\begin{tabular}{|c|c|}
\hline Intervention & Date of implementation \\
\hline \multicolumn{2}{|l|}{ Patient-related } \\
\hline Patient symptom screening twice per shift ${ }^{\star} \dagger$ & June 4, 2020 \\
\hline Creation of a dedicated COVID-19 unit & July 5, 2020 \\
\hline $\begin{array}{l}\text { Development and implementation of an approach to managing patients with unrecognized } \\
\text { SARS-CoV-2 infection who wandered }\end{array}$ & July 5, 2020 \\
\hline $\begin{array}{l}\text { Dual sign-off requirement for the removal of contact and droplet precautions from patients with } \\
\text { suspected COVID-19 who tested negative }\end{array}$ & Aug. 9, 2020 \\
\hline \multicolumn{2}{|l|}{ Staff-related } \\
\hline Training and deployment of PPE coaches & June 30, 2020 \\
\hline Training and deployment of PPE auditors & June 30, 2020 \\
\hline Addition of continuous eye protection to continuous masking for all staff with patient interactions & July 5,2020 \\
\hline Fit-to-work screening online questionnaire twice per shift & July 8,2020 \\
\hline Break room and locker room sign-ins and physical distancing in break areas & July 12,2020 \\
\hline \multicolumn{2}{|c|}{$\begin{array}{l}\text { Note: } \mathrm{PCR}=\text { polymerase chain reaction, } \mathrm{PPE}=\text { personal protective equipment. } \\
\text { "Evaluation for new or worsening symptoms of core symptoms (cough; fever }\left[>37.8^{\circ} \mathrm{C}\right] \text {, chills or rigours; shortness of breath; increased oxygen requirement; decreased } \\
\text { oxygen saturation; difficulty breathing; sore throat or painful swallowing; runny nose or nasal congestion; loss of or change to sense of smell or taste), gastrointestinal } \\
\text { symptoms (vomiting or diarrhea), or expanded symptoms (headache; muscle or joint pain; fatigue; extreme exhaustion; nausea; sudden loss of appetite; conjunctivitis or red } \\
\text { eye; conjunctival edema; additional COVID-19 symptoms at clinician's discretion) and altered mental status. Identification of any of these resulted in a review with the } \\
\text { attending physician to discuss ordering a new or repeat SARS-CoV-2 nasopharyngeal swab PCR test. } \\
\text { tPatients were given education on how to use the surgical mask and hand sanitizer by their nursing teams. Extra masks were provided upon patient request. Hand sanitizer } \\
\text { was not provided to patients where it was felt that it may pose a health hazard; instead, these patients were asked to perform hand hygiene with assistance whenever } \\
\text { interacting with health care staff. } \\
\text { fThe dual signature requirement for the removal of precautions included the patient's most responsible physician (or nurse practitioner) and a senior nurse in charge on the unit. }\end{array}$} \\
\hline
\end{tabular}

\section{Ethics approval}

Analysis and presentation of the data from this outbreak was approved by the Health Research Ethics Board at the University of Alberta (study identifier Pro00103046).

\section{Results}

The COVID-19 outbreak included 58 patient, staff and visitor cases (Table 2; Figure 1). Two-thirds of all cases were in women; 5 of $58(9 \%)$ and 53 of $58(91 \%)$ of cases were categorized as community-acquired and hospital-acquired, respectively. Over one-quarter $(16 / 58,28 \%)$ were asymptomatic at diagnosis. One health care worker required admission to the intensive care unit after exposure to a wandering inpatient with dementia and asymptomatic, undetected SARS-CoV-2 infection. The outbreak was associated with 11 inpatient deaths (19\%).

Thirteen secondary cases linked to the outbreak were household contacts of patients, staff or visitors (Table 2). One-third of the 25 staff cases were a result of transmission in small break rooms or locker rooms.

\section{Chronology of the outbreak}

On June 21, 2020, a COVID-19 outbreak was declared when IPC was notified of 2 confirmed cases of SARS-CoV-2 infection on a single patient unit: a staff member and a patient who was admitted June 10, 2020. A lack of an epidemiologic link between these 2 cases raised concerns about an undiagnosed source of SARS-CoV-2 on the unit.
Investigation found that the patient had symptoms consistent with COVID-19 upon admission but had tested negative, and isolation precautions were discontinued prematurely. When the patient's symptoms did not improve, repeat testing on June 20, 2020, confirmed a diagnosis of COVID-19.

On the following day (June 21, 2020), 5 (17\%) of the 29 patients remaining on the unit were diagnosed with SARS$\mathrm{CoV}-2$ infection on prevalence screening. Repeat screening on June 24, 2020, identified another case (1/13, 8\%). Additionally, contact tracing showed that some roommates of newly identified cases had been transferred to the onsite rehabilitation unit; prevalence screening in that unit on June 25, 2020 , found that another 3 of 18 patients (17\%) were positive for SARS-CoV-2 infection (Appendix 1, Table S1).

Prevalence screening of involved units continued every 72 hours (Appendix 1, Table S1). On July 7, 2020, 7 of 62 patients (11\%) tested positive for SARS-CoV-2 infection. In addition, a large number of staff were mandated to quarantine for 14 days after being identified as having an exposure to patients or staff who tested positive for SARS-CoV-2. In the first 4 weeks of the outbreak, 21 patient cases were identified in this manner.

In view of the outbreak progression and staff shortages, the hospital was closed to new admissions and transfers on July 8 , 2020, after consultation with local public health and regional health authorities. ${ }^{27}$ Patients were discharged home when possible, and remaining inpatients received all necessary medical, surgical and critical care services. The emergency department stayed open for walk-in patients only; any who required admission were transferred to another facility. 


\begin{tabular}{|c|c|}
\hline \multicolumn{2}{|c|}{$\begin{array}{l}\text { Table 2: Demographic characteristics of primary and } \\
\text { secondary outbreak cases }\end{array}$} \\
\hline Variable & No. $(\%)^{*}$ \\
\hline \multicolumn{2}{|l|}{ Primary cases $(n=58)$} \\
\hline \multicolumn{2}{|l|}{ Age, yr, mean (range) } \\
\hline Overall & $62(19-97)$ \\
\hline Patients & $79(24-97)$ \\
\hline Visitors & $75(71-78)$ \\
\hline Staff & $41(19-62)$ \\
\hline \multicolumn{2}{|l|}{ Case classification } \\
\hline Patient & $31(54)$ \\
\hline Visitor & $2(3)$ \\
\hline Staff & $25(43)$ \\
\hline \multicolumn{2}{|l|}{ Sex, female } \\
\hline Overall & $38(66)$ \\
\hline Patients & $14(45)$ \\
\hline Visitors & $2(100)$ \\
\hline Staff & $22(88)$ \\
\hline \multicolumn{2}{|l|}{ Acquisition } \\
\hline Community & $5(9)$ \\
\hline Hospital & $53(91)$ \\
\hline \multicolumn{2}{|l|}{ Symptoms at time of diagnosis } \\
\hline Symptomatic & $42(72)$ \\
\hline Asymptomatic & $16(28)$ \\
\hline \multicolumn{2}{|l|}{ Staff role } \\
\hline Nurse or student nurse & 19 \\
\hline Health care aide & 1 \\
\hline Housekeeping staff & 1 \\
\hline Pharmacist & 1 \\
\hline Physician & 1 \\
\hline Security guard & 1 \\
\hline Service attendant & 1 \\
\hline Critical care admissions & $1(2) \dagger$ \\
\hline Deaths & $11(19) \ddagger$ \\
\hline \multicolumn{2}{|c|}{ Secondary cases linked to the outbreak $(n=13)$} \\
\hline Age, yr, mean (range) & $39(3-84)$ \\
\hline Sex, female & $2(15)$ \\
\hline \multicolumn{2}{|l|}{ Type of contact } \\
\hline Household contact of patient & $4(31)$ \\
\hline Household contact of visitor & $1(8)$ \\
\hline Household contact of staff & $8(61)$ \\
\hline \multicolumn{2}{|c|}{$\begin{array}{l}\text { *Except where noted otherwise. } \\
\text { †Occurred in } 1 \text { staff member who contracted the infection from a patient with } \\
\text { underlying dementia and wandering behaviour. } \\
\text { †All deaths occurred in the hospital inpatient group. No deaths occurred in staf } \\
\text { members or visitors linked to the outbreak. }\end{array}$} \\
\hline
\end{tabular}

\section{Staff prevalence screening}

Voluntary onsite SARS-CoV-2 testing of asymptomatic staff identified 22 cases (Appendix 1, Table S2). Investigation found several potential sources of SARS-CoV-2 exposure among staff: unprotected exposure to a wandering patient with undiagnosed SARS-CoV-2 infection, inappropriate PPE use with unrecognized symptomatic patients who eventually tested positive for SARS-CoV-2, inappropriate PPE use in shared staff settings, PPE breaches and exposure to other staff members who came to work displaying symptoms and were later confirmed to be positive for SARS-CoV-2.

\section{Transmission patterns}

Detailed contact tracing showed that the outbreak consisted of 2 main clusters spread over 6 units (Appendix 1, Figure S1 and Table S3). Mapping confirmed extensive chains of transmission, with multiple reasons for cluster propagation. In clusters $\mathrm{A}$ and $\mathrm{B}$, transmission was secondary to inadequate use of PPE by staff, spread in enclosed staffing areas (e.g., break rooms), removal of isolation precautions without confirming SARS-CoV-2 test results (1 patient, cluster A) and failure to evaluate the need for repeat testing (2 patients, cluster A). Transmission in clusters A and B was compounded by the high proportion of multi-bed rooms in our facility, with attack rates of $100 \%$ once a roommate had acquired SARS-CoV-2; 9 multi-bed rooms were affected during this outbreak.

Transfer of undiagnosed patients to other units and missed opportunities for testing as a result of discrepancies in patient symptom charting were also noted across both clusters. Thus, isolation precautions were removed prematurely in 3 patients who were later diagnosed with SARS-CoV-2 infection on prevalence screening and determined to be the index cases of cluster A. As a result of this finding, the outbreak response team implemented a 2-person sign-off protocol to discontinue isolation precautions in any patient with suspected SARS$\mathrm{CoV}-2$ infection - an intervention that required clinical reevaluation by the most responsible health care provider and another regulated staff member.

Wandering or aggressive behaviours in patients with dementia and unrecognized SARS-CoV-2 infection contributed to further transmission among patients and staff in both clusters. Two cases of SARS-CoV infection (unknown cluster in Appendix 1, Figure S1) were staff members who worked and interacted with patients and other staff on multiple units, but clear transmission pathways were not determined, and thus these cases could not be attributed with confidence to either cluster.

\section{Interpretation}

We have described a large hospital outbreak of COVID-19 in Edmonton that affected a total of 58 patients, staff and visitors; more than $90 \%$ of cases were hospital-acquired. The overall case fatality rate was high (19\%), occurring entirely among inpatients. The outbreak was eventually brought under control as a result of hospital closure combined with investigation, implementation and enforcement of a series of measures to decrease further transmission of SARS-CoV-2.

Multiple clusters have been reported in previous COVID19 outbreaks. ${ }^{30-33}$ This outbreak comprised 2 primary clusters across multiple patient units. Investigation for multiple clusters is important to elucidate individual transmission events 
A

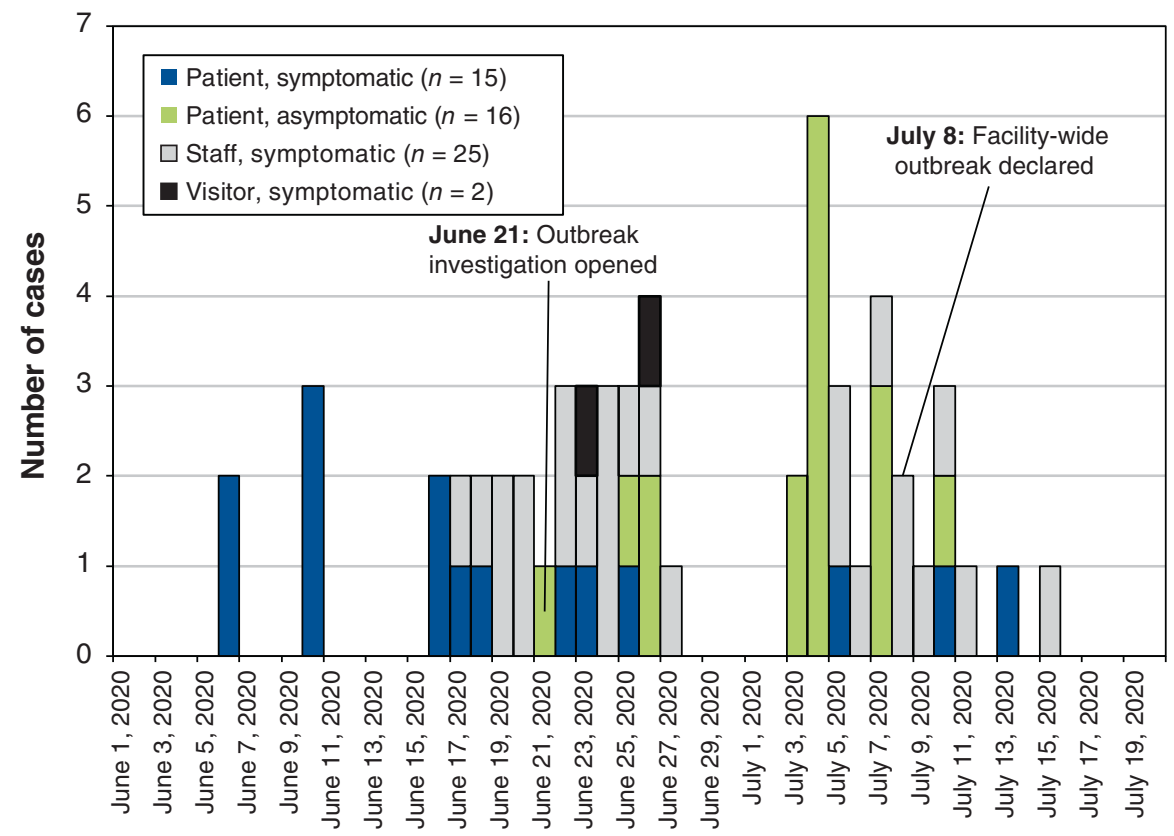

Onset date

B

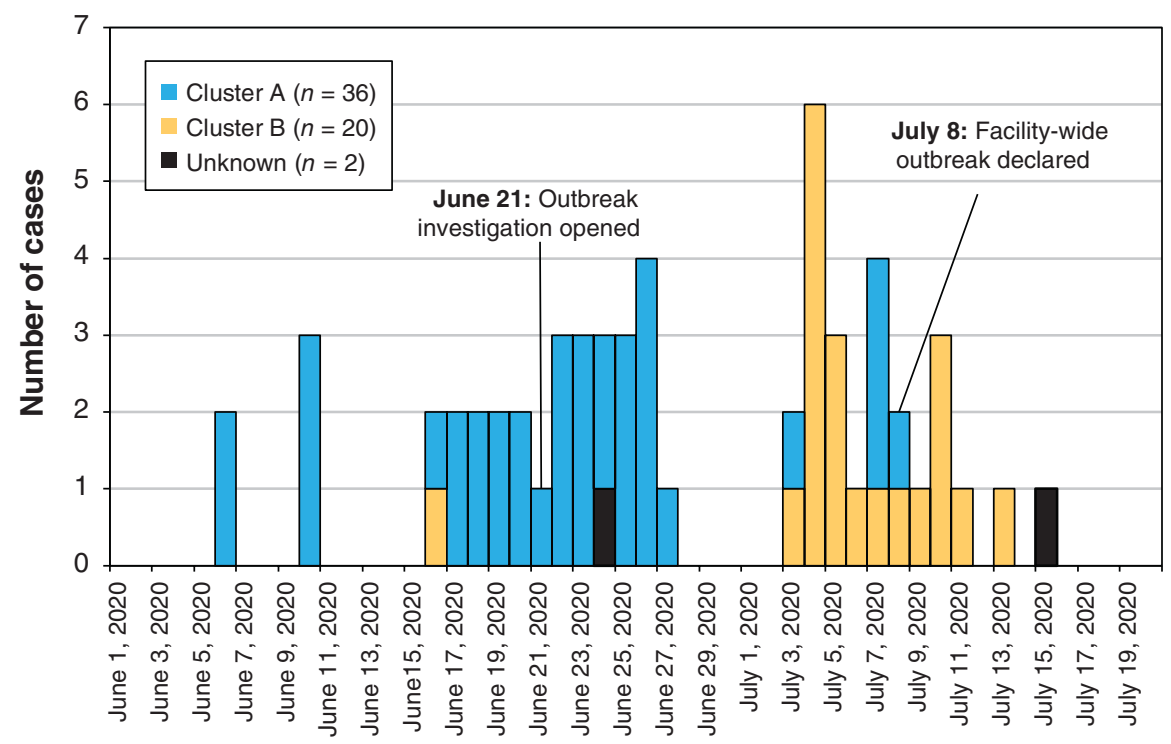

Onset date

Figure 1: Epidemiologic curve demonstrating the timeline of outbreak cases by symptom onset date, stratified by (A) case category and (B) cluster ( $n=58$ for both curves). For those who were asymptomatic, the date of a positive specimen collection was used as the onset date. 
and interrupt chains of transmission. ${ }^{34}$ The clusters in this outbreak were driven by several factors, as outlined in the results above. Clusters were generally linked by 1 or 2 cases with unknown transmission across units, especially during the asymptomatic phase of infection. ${ }^{35,36}$

Our findings highlight the increased risk of SARS-CoV-2 transmission among staff in small enclosed spaces, such as break rooms and work rooms. Similar findings have also been reported in different health care scenarios. ${ }^{37,38} \mathrm{~A}$ valuable approach used in mitigating this risk for staff in our hospital included strict adherence to PPE use in shared areas, physical distancing for food and hydration breaks, and the provision of surveillance screening for asymptomatic staff members to identify staff cases at an early stage, an approach that has been described in other outbreak reports. ${ }^{37}$ Screening of asymptomatic staff identified 22 of 25 (88\%) staff cases during this outbreak.

A principal challenge was reliance on a single negative test to rule out a diagnosis of COVID-19 in patients who continued to have compatible symptoms. False negative rates can range from $2.2 \%$ to $17 \%,{ }^{16,26,39}$ and can be attributed to suboptimal specimen collection, testing early in the course of infection, variability in viral shedding, low viral load and variation in specimen types. ${ }^{16,26}$ After the investigation, widespread education about the consequences of COVID-19 misdiagnosis was provided, emphasizing the need to re-evaluate patients with persistent or unexplained symptoms for COVID-19, especially if they could not communicate their symptoms. ${ }^{40-42}$

Patients with SARS-CoV-2 infection and dementia complicated by wandering and aggression pose a high risk of transmitting infection to other patients and health care workers. ${ }^{43}$ Symptoms can be challenging to identify and diagnostic specimens difficult to obtain, leading to delays in diagnosis. ${ }^{44}$ Furthermore, this patient population can be unable to understand, remember and follow restrictive health measures such as isolation, hand hygiene and continuous masking. ${ }^{45}$ Delayed evaluation and detection of SARSCoV-2 infection in 2 such patients resulted in transmission to 10 patients and staff. Similar concerns have been reported in other outbreaks; ${ }^{10}$ a sensitive and multidisciplinary approach is required. The outbreak response team, in collaboration with the local health authority, rapidly developed a set of guidelines that were implemented mid-outbreak (July 5, 2020) to help prevent and manage behaviours in this group of patients (Appendix 1, Methods S2).

Prevalence screening for SARS-CoV-2 infection on outbreak units was an invaluable outbreak management tool and containment measure. Screening patients every 72 hours helped to identify patients in a presymptomatic phase, so that they could be immediately moved to a COVID-19 isolation unit and minimize further transmission. Universal prevalence screening in an outbreak setting is increasingly supported by literature showing that the screening of symptomatic patients in an outbreak alone is insufficient for preventing further nosocomial spread. ${ }^{10,33,46,47}$ Data from a single outbreak noted that up to $95 \%$ of transmission was suspected to occur during the asymptomatic period. ${ }^{36}$ This practice is also beneficial for outbreaks involving patients for whom reliable symptom evaluation is challenging because of underlying behaviour or functional variables. ${ }^{48}$ Limitations of this practice should be considered in terms of availability of high-volume polymerase chain reaction testing and quick turnaround time, so that results can be acted upon urgently.

\section{Limitations}

Several limitations in this outbreak description should be noted. The Misericordia Community Hospital is an older facility compared to many hospitals in the region; although the building is actively maintained, its age posed a number of architecture-related challenges. Most inpatient spaces are 4-bed rooms, which led to higher potential for transmission and outbreak compared to newer centres with more single rooms and patient isolation spaces. ${ }^{49}$ The hospital's heating, ventilation and air conditioning system was evaluated by the facility management team, who advised that it was maintained and in working order for the type of system originally installed. However, it must be acknowledged that newer facilities likely have improved systems.

This outbreak occurred at a time when prepandemic planning had been implemented, including cancellation of elective surgical procedures, which afforded the hospital some empty surgical units to serve as decanting space to manage outbreaks. This outbreak may have unfolded differently if there had been limited space for the creation of a COVID-19 unit.

Hospital-acquired cases of SARS-CoV-2 infection may have been underestimated if people were exposed to highly contagious sources, because cases with shorter incubation periods than the median used for classification have been reported. ${ }^{50}$

Because several IPC and OHS measures were implemented in a short time period, we could not systematically evaluate the individual contribution of each intervention in resolving the outbreak. However, we were able to demonstrate that the implementation of PPE coaches and auditors decreased errors related to PPE by an average of 7\%, with a $23 \%$ decrease in observed PPE doffing errors. ${ }^{51}$

\section{Conclusion}

Although SARS-CoV-2 outbreaks may appear to be singleevent transmissions, many comprise multiple transmission clusters, as in the large hospital outbreak we described. Given the prolonged incubation period of SARS-CoV-2, a moderate degree of virus transmission has often occurred at the time of outbreak detection. Furthermore, the challenge of evaluating the potential for asymptomatic spread from unique populations, such as those with dementia and wandering behaviours, is high. With multipronged bundled approaches, combined with universal prevalence screening and aggressive contact tracing, outbreaks can be rapidly brought under control but require vigilance to ensure the detection of delayed cases. 


\section{References}

1. Alimohamadi Y, Taghdir M, Sepandi M. Estimate of the basic reproduction number for COVID-19: a systematic review and meta-analysis. 7 Prev Med Public Health 2020; 53:151-7.

2. Zhou Q, Gao Y, Wang X, et al. Nosocomial infections among patients with COVID-19, SARS and MERS: a rapid review and meta-analysis. Ann Transl Med 2020;8:629.

3. Vanhems P, Saadatian-Elahi M, Chuzeville M, et al. Rapid nosocomial spread of SARS-CoV-2 in a French geriatric unit. Infect Control Hosp Epidemiol 2020;41:866-7.

4. Biernat MM, Zinczuk A, Biernat P, et al. Nosocomial outbreak of SARSCoV-2 infection in a haematological unit - high mortality rate in infected patients with haematologic malignancies. F Clin Virol 2020;130:104574.

5. Rickman HM, Rampling T, Shaw K, et al. Nosocomial transmission of COVID-19: a retrospective study of 66 hospital-acquired cases in a London teaching hospital. Clin Infect Dis 2021;72:690-3.

6. Remuzzi A, Remuzzi G. COVID-19 and Italy: What next? Lancet 2020;395: 1225-8.

7. Kluytmans-van den Bergh MFQ, Buiting AGM, Pas SD, et al. Prevalence and clinical presentation of health care workers with symptoms of coronavirus disease 2019 in 2 Dutch hospitals during an early phase of the pandemic. 7AMA Netw Open 2020;3:e209673.

8. Rhee C, Baker M, Vaidya V, et al. Incidence of nosocomial COVID-19 in patients hospitalized at a large US academic medical center. $7 A M A$ Netw Open 2020;3:e2020498.

9. Turale S, Meechamnan C, Kunaviktikul W. Challenging times: ethics, nursing and the COVID-19 pandemic. Int Nurs Rev 2020;67:164-7.

10. Höring S, Fussen R, Neusser J, et al. Management of a hospital-wide COVID19 outbreak affecting patients and healthcare workers. SN Compr Clin Med 2020;Oct 26 [Epub ahead of print]. doi: 10.1007/s42399-020-00597-2.

11. Mungmunpuntipantip R, Wiwanitkit V. A coronavirus disease 2019 (COVID-19) outbreak in a hospital and hospital closure: a note. Infect Control Hosp Epidemiol 2020;41:1475-6.

12. Lawrence AC, Burke C, Brister J, et al. Evaluating the impact of hospital closure on local emergency department operations. RI Med 7 (2013) 2019;102:37-42

13. Walker KO, Calmes D, Hanna N, et al. The impact of public hospital closure on medical and residency education: implications and recommendations. 7 Natl Med Assoc 2008;100:1377-83.

14. Pabbaraju K, Wong A, Douesnard M, et al. Development and validation of reverse transcriptase-PCR assays for the testing of SARS-CoV-2. 7 Virol Methods 2021;293:114151.

15. Pabbaraju K, Wong AA, Douesnard M, et al. A public health laboratory response to the pandemic. 7 Clin Microbiol 2020;58:e01110-20.

16. Kanji JN, Zelyas N, MacDonald C, et al. False negative rate of COVID-19 PCR testing: a discordant testing analysis. Virol f 2021;18:13.

17. Lauer SA, Grantz KH, Bi Q, et al. The incubation period of coronavirus disease 2019 (COVID-19) from publicly reported confirmed cases: estimation and application. Ann Intern Med 2020;172:577-82.

18. COVID-19 provincial surveillance protocol. Edmonton: Provincial Infection Prevention \& Control Surveillance Committee (Alberta Health Services); 2020. Available: www.albertahealthservices.ca/assets/healthinfo/ipc/hi-ipc-sr -covid-surveillance-protocol.pdf (accessed 2021 Aug. 1).

19. Public bealth management of cases and contacts associated with COVID-19. Ottawa: Government of Canada; 2021.

20. Symptoms and testing: COVID-19 testing is available to all Albertans with symptoms, close contacts of confirmed cases and those linked to an outbreak. Edmonton: Government of Alberta; 2021. Available: www.alberta.ca/covid-19-testing-in -alberta.aspx (accessed 2020 Dec. 10).

21. AHS acute care COVID-19 expanded testing algorithm. Edmonton: Alberta Health Services; 2021. Available: www.albertahealthservices.ca/assets/info/ ppih/if-ppih-covid-19-expanded-testing.pdf (accessed 2021 Aug. 1).

22. COVID-19: main modes of transmission. Ottawa: Government of Canada; 2021. Available: www.canada.ca/en/public-health/services/diseases/2019-novel -coronavirus-infection/health-professionals/main-modes-transmission.html (accessed 2021 Feb. 27).

23. Aerosol-generating medical procedure guidance tool: COVID-19. Edmonton: Alberta Health Services; 2021. Available: www.albertahealthservices.ca/ topics/Page17091.aspx (accessed 2021 Aug. 5.)

24. Discontinuation of transmission-based precautions and disposition of patients with COVID-19 in bealthcare settings (interim guidance). Atlanta: Centers for Disease Control and Prevention; 2021. Available: www.cdc.gov/coronavirus /2019-ncov/hcp/disposition-hospitalized-patients.html\#: :text=Changes\%20 to $\% 20$ more $\% 20$ closely $\% 20$ align,20\%20days \%20after\%20symptom\%20onset (accessed 2020 Dec. 10).

25. Point of care risk assessment. Edmonton: Alberta Health Services; 2018. Available: www.albertahealthservices.ca/ipc/hi-ipc-routine-practices-algorithm-cc. pdf (accessed 2021 Aug. 27).
26. Dugdale CM, Anahtar MN, Chiosi JJ, et al. Clinical, laboratory, and radiologic characteristics of patients with initial false-negative SARS-CoV-2 nucleic acid amplification test results. Open Forum Infect Dis 2020;8:ofaa559.

27. Incident Command System Alberta. Edmonton: Government of Alberta; 2021. Available: www.alberta.ca/incident-command-system-alberta.aspx (accessed 2021 Feb. 12)

28. Bastian MHS, Jacomy M. Gephi: an open source software for exploring and manipulating networks. In: Proceedings from the Third International AAAI Conference on Weblogs and Social Media; 2009 May 17-20; San Jose (CA). Burnaby (BC): PKP Publishing Services; 2009;3:361-2. Available: https://ojs.aaai.org/ index.php/ICWSM/article/view/13937 (accessed 2020 Nov. 2).

29. Jacomy M, Venturini T, Heymann S, et al. ForceAtlas2, a continuous graph layout algorithm for handy network visualization designed for the Gephi software. PLoS One 2014;9:e98679.

30. Richterman A, Meyerowitz EA, Cevik M. Hospital-acquired SARS-CoV-2 infection: lessons for public health. 7AMA 2020;324:2155-6.

31. Snell L, Fisher C, Taj U, et al. Combined epidemiological and genomic analysis of nosocomial SARS-CoV-2 transmission identifies community social distancing as the dominant intervention reducing outbreaks [preprint]. medRxiv 2020 Nov. 18. doi: https://doi.org/10.1101/2020.11.17.20232827.

32. Scientific brief: transmission of SARS-CoV-2: implications for infection prevention precautions. Geneva: World Health Organization; 2020. Available: www.who. $\mathrm{int} /$ news-room/commentaries/detail/transmission-of-sars-cov-2-implications -for-infection-prevention-precautions (accessed 2020 Dec. 30).

33. Klompas M, Baker MA, Rhee C, et al. A SARS-CoV-2 cluster in an acute care hospital. Ann Intern Med 202 1;174:794-802.

34. Price JR, Mookerjee S, Dyakova E, et al. Development and delivery of a realtime hospital-onset COVID-19 surveillance system using network analysis. Clin Infect Dis 2021;72:82-9.

35. Yong SEF, Anderson DE, Wei WE, et al. Connecting clusters of COVID19: an epidemiological and serological investigation. Lancet Infect Dis 2020;20:809-15.

36. Harada S, Uno S, Ando T, et al. Control of a nosocomial outbreak of COVID-19 in a university hospital. Open Forum Infect Dis 2020;7:ofaa512.

37. Celebi G, Piskin N, Celik Bekleviç A, et al. Specific risk factors for SARS $\mathrm{CoV}-2$ transmission among health care workers in a university hospital. $A m \mathcal{F}$ Infect Control 2020;48:1225-30.

38. Ariza-Heredia EJ, Frenzel E, Cantu S, et al. Surveillance and identification of clusters of healthcare workers with coronavirus disease 2019 (COVID-19): multidimensional interventions at a comprehensive cancer center. Infect Control Hosp Epidemiol 202 1;42:797-802.

39. Green DA, Zucker J, Westblade LF, et al. Clinical performance of SARSCoV-2 molecular tests. 7 Clin Microbiol 2020;58:e00995-20.

40. The Infectious Diseases Society of America guidelines on the diagnosis of COVID-19: molecular diagnostic testing. Arlington: Infectious Diseases Society of America; 2021. Available: www.idsociety.org/practice-guideline/covid-19-guideline -diagnostics/ (accessed 2020 Dec. 3).

41. Diagnostic testing for SARS-CoV-2. Geneva: World Health Organization; 2020. Available: www.who.int/publications/i/item/diagnostic-testing-for -sars-cov-2 (accessed 2020 Sep. 19).

42. Budhram B, Kobza AO, Mohammed N. Misdiagnosis related to premature diagnostic closure during the COVID-19 pandemic. CMA7 2020;192:E1129-31.

43. Numbers K, Brodaty H. The effects of the COVID-19 pandemic on people with dementia. Nat Rev Neurol 2021;17:69-70.

44. Fahed M, Barron GC, Steffens DC. Ethical and logistical considerations of caring for older adults on inpatient psychiatry during the COVID-19 pandemic. Am 7 Geriatr Psychiatry 2020;28:829-34.

45. Nkodo J-A, Camus V, Fougère B. Ethical issues in the management of patients with behavioral and psychological symptoms of dementia during COVID-19 containment: examples from institutions in France. Am 7 Geriatr Psychiatry 2020;28:1332-3.

46. Eckardt P, Guran R, Hennemyre J, et al. Hospital affiliated long term care facility COVID-19 containment strategy by using prevalence testing and infection control best practices. Am $\mathcal{F}$ Infect Control 2020;48:1552-5.

47. Hatfield KM, Reddy SC, Forsberg K, et al. Facility-wide testing for SARSCoV-2 in nursing homes - seven U.S. jurisdictions, March-June 2020. MMWR Morb Mortal Wkly Rep 2020;69:1095-9.

48. Birgand G, Blanckaert K, Deschanvres C, et al. Testing strategies for the control of COVID-19 in nursing homes: Universal or targeted screening? 7 Infect 2021;82:159-98.

49. Karan A, Klompas M, Tucker R, et al. The risk of SARS-CoV-2 transmission from patients with undiagnosed COVID-19 to roommates in a large academic medical center. Clin Infect Dis 2021 Jun 18;ciab564 Epub ahead of print]. doi: 10.1093/cid/ciab564.

50. Lai $\mathrm{C}, \mathrm{Yu} \mathrm{R}, \mathrm{Wang} \mathrm{M}$, et al. Shorter incubation period is associated with severe disease progression in patients with COVID-19. Virulence 2020;11:1443-52.

51. Picard C, Edlund M, Keddie C, et al. The effects of trained observers (dofficers) and audits during a facility-wide COVID-19 outbreak: a mixed-methods quality improvement analysis. Am 7 Infect Control 2021;49:1136-41. 
Affiliations: Division of Infectious Diseases, Department of Medicine (Kanji, Boychuk, Boyington, Smith), University of Alberta; Covenant Health (Kanji, Boychuk, Boyington, Turay, Kobelsky, Doroshuk, Choo, Jacka, Roberts, Leighton, Black); Canadian Public Health Service, Public Health Agency of Canada (Chan); Division of Preventive Medicine, Faculty of Medicine and Dentistry (Sikora), University of Alberta; Medical Officer of Health (Edmonton Zone), (Sikora) Alberta Health Services; Department of Obstetrics and Gynecology, Faculty of Medicine and Dentistry (Black), University of Alberta, Edmonton, Alta. Note: Dr. J.N. Kanji is now with the Division of Infectious Diseases, Department of Medicine and Department of Pathology and Laboratory Medicine, University of Calgary, Calgary, Alta.

Contributors: Jamil Kanji contributed to conceptualization, data curation, formal analysis, validation and writing the original draft. Elaine Chan contributed to data curation, methodology, formal analysis, software and visualization. Lesia Boychuk contributed to project administration and supervision. Curtiss Boyington contributed to project administration, investigation and supervision. Sebora Turay and Philana Choo contributed to investigation and formal analysis. Melissa Koblesky, Carolyn Doroshuk, Susan Jacka and Karen Leighton contributed to investigation. Erin Roberts contributed to investigation, supervision and validation. Stephanie Smith and Christopher Sikora contributed to supervision and validation. Robert Black contributed to supervision, project administration and validation. All of the authors revised the manuscript critically for important intellectual content, gave final approval of the version to be published and agreed to be accountable for all aspects of the work.

Funding: This work did not receive any specific grant from funding agencies in the public, commercial or not-for-profit sectors.

Content licence: This is an Open Access article distributed in accordance with the terms of the Creative Commons Attribution (CC BY-NC-ND 4.0) licence, which permits use, distribution and reproduction in any medium, provided that the original publication is properly cited, the use is noncommercial (i.e., research or educational use), and no modifications or adaptations are made. See: https://creativecommons.org/licenses/ by-nc-nd/4.0/

Data sharing: The data sets used and analyzed for the current study are available upon reasonable request from the corresponding author.

Acknowledgements: The authors would like to acknowledge Dr. Marcia Johnson and Alberta Health for their assistance with outbreak analysis and epidemiologic support. Furthermore, Dr. Johnson's support with supervision, drafting, and review of the initial outbreak report was instrumental. They would also like to acknowledge the health care workers of the Misericordia Hospital for their dedication, hard work, vigilance, and persistence during the COVID-19 pandemic as well as the Public Health Laboratory (Alberta Precision Laboratories) for their exceptional testing ability and pandemic response innovation.

Supplemental information: For reviewer comments and the original submission of this manuscript, please see www.cmajopen.ca/content /10/1/E137/suppl/DC1. 